Revue d'histoire de l'enfance « irrégulière »

Le Temps de l'histoire

Hors-série | 2007

Pages d'histoire, la protection judiciaire des mineurs, $\mathrm{XIX} \mathrm{X}^{\mathrm{e}} \mathrm{XX} \mathrm{X}^{\mathrm{e}}$ siècles

\title{
Tableau chronologique Justice-Santé-Education
} (1927-1948)

Jacques Bourquin et Michel Chauvière

\section{(2) OpenEdition \\ Journals}

Édition électronique

URL : http://journals.openedition.org/rhei/3117

DOI : 10.4000/rhei.3117

ISBN : 978-2-7535-1647-2

ISSN : $1777-540 \mathrm{X}$

Éditeur

Presses universitaires de Rennes

Édition imprimée

Date de publication : 1 juin 2007

Pagination : 311-319

ISSN : 1287-2431

Référence électronique

Jacques Bourquin et Michel Chauvière, «Tableau chronologique Justice-Santé-Education

(1927-1948) », Revue d'histoire de l'enfance « irrégulière » [En ligne], Hors-série | 2007, mis en ligne le 01

février 2010, consulté le 01 mai 2019. URL : http://journals.openedition.org/rhei/3117 ; DOI : 10.4000/ rhei.3117 


\section{Éléments de chronologie comparative Justice-Santé-Éducation nationale $1930-1947^{(*)}$}

Si la période 1880-1914 est généralement tenue pour innovante et décisive du point de vue de l'intérêt de l'enfant dans le droit, une seconde période créative, au plan législatif, réglementaire tout autant qu'au plan institutionnel et pratique, court de la fin des années 1920 à la fin des années 1940. C'est cette double décennie, qui connaît la crise des années 1930, le Front populaire, la deuxième guerre mondiale et le régime de Vichy, la Libération et la reconstruction nationale, que nous avons choisie pour ces chronologies parallèles. On pourrait dire qu'il s'agit de la deuxième vague réformiste en faveur de l'enfance.

Trois acteurs publics sont maintenant à considérer.

Le ministère de la Justice est de tous le plus ancien et sans doute aussi le plus légitime en matière d'enfance délinquante et plus largement d'ordre public. C'est en son sein que se sont lentement construits le droit des mineurs, la défense sociale, l'éducation surveillée en attendant la protection judiciaire de la jeunesse.

Le ministère de l'Éducation nationale, ainsi nommé depuis 1932, se définit par sa mission civilisatrice, relativement hégémonique; mais celle-ci bute tout à la fois sur l'existence de courants pédagogiques alternatifs, catholiques ou simplement proactifs, sur la découverte de la " débilité intellectuelle " et, corollairement, la révélation d'une indiscipline du comportement à l'école, tous phénomènes qui, cumulés, ruinent les bases du projet scolaire. C'est un acteur très légitime mais, à ces dates, il reste relativement marginal dans le domaine des enfances difficiles.

Enfin le ministère de la Santé publique; il n'a guère de compétences en début de période (hormis l'assistance publique et le contrôle sanitaire des établissements), mais bénéficie dès le régime de Vichy d'une valorisation inattendue, au nom d'alliances conjoncturelles familialistes et contre l'école publi-
(*) Texte rédigé par

Jacques Bourquin et

Michel Chauvière, publié

dans le Temps de

l'histoire, $\mathrm{n}^{\circ} 1$, février 1998.

Éléments de chronologie comparative... / p. 311 à 319 
(1) Psychiatre lyonnais.

que; c'est lui qui va offrir toute sa place à la modernité médico-psychiatrique dans le diagnostic comme dans le traitement de l'enfance « irrégulière ». La Libération ne laissera toutefois pas la Santé phagocyter le secteur de la Justice des mineurs.

Si les poids politiques et les responsabilités pratiques respectives de ces trois acteurs publics sont inégaux, la recherche d'une introuvable coordination hante également la période. Elle se fait par principe au bénéfice de l'Éducation nationale alliée à la Justice sous le Front populaire, mais ses retombées sont limitées. Elle bascule concrètement et très officiellement au bénéfice du ministère de la Santé en 1941, puis elle est concrètement mise en œuvre par son appendice, le Commissariat général à la famille, pendant toute la période de la guerre et du régime de Vichy. Dans l'après-guerre, l'Éducation nationale retrouve sa place légitime et l'Éducation surveillée s'ancre définitivement au ministère de la Justice, rendant plus problématique encore toute idée de coordination interministérielle ou d'action concertée pour la protection de l'enfance.

Comme on le voit, la période est riche en innovations législatives et institutionnelles de toutes sortes, qui correspondent aussi à des tensions entre des référentiels contradictoires pour l'action publique, inspirés de moments politiques discontinus. Ces données révèlent également la difficile unité du champ de l'enfance inadaptée et les enjeux concrets de cette nouvelle politique publique. Au mot de Dechaume, ${ }^{(1)}$ " l'enfance inadaptée est une, la délinquance n’est qu'un accident ", répond tout à la fois le principe de l'irréductibilité du délit, malgré l'orientation sociale prônée par l'ordonnance du 2 février 1945 sur l'enfance délinquante, et l'évocation incantatoire et itérative, dès la Libération, d'une réforme en profondeur de l'Éducation nationale qui tarirait définitivement les causes de l'échec et de l'inadaptation scolaires.

Finalement, cette deuxième vague réformiste a créé les infrastructures pratiques de l'actuel secteur de l'éducation spéciale, spécialisée ou surveillée, sans parvenir à agir de manière significative sur la nature des phénomènes visés. 
Nous citons en préalable quatre lois fondatrices qui précèdent la période :

- Loi du 24 juillet 1889, sur la protection des enfants maltraités et moralement abandonnés.

- Loi du 28 juin 1904, relative à l'éducation des pupilles difficiles et vicieux de l'Assistance publique (création des classes et écoles autonomes de perfectionnement).

- Loi du 15 avril 1909, relative à l'instruction publique des enfants déficients.

- Loi du 12 juillet 1912, sur les tribunaux pour enfants et adolescents et la liberté surveillée. 


\begin{tabular}{|c|c|c|c|c|}
\hline Année & Législation & Justice & Santé & Éducation nationale \\
\hline 1927 & $\begin{array}{l}\text { Décret-loi du } \\
31 \text { décembre }\end{array}$ & $\begin{array}{l}\text { création des maisons d'éducation } \\
\text { surveillée }\end{array}$ & & \\
\hline 1928 & $\begin{array}{l}\text { Décret du } \\
26 \text { février }\end{array}$ & & & $\begin{array}{l}\text { création de l'Institut national } \\
\text { d'orientation professionnelle }\end{array}$ \\
\hline 1930 & $\begin{array}{l}\text { Arrêté du } \\
10 \text { avril }\end{array}$ & $\begin{array}{l}\text { règlement général des maisons } \\
\text { d'éducation surveillée }\end{array}$ & & \\
\hline \multirow[t]{3}{*}{1932} & $\begin{array}{l}\text { Décret du } \\
12 \text { janvier }\end{array}$ & & $\begin{array}{l}\text { brevet de capacité professionnelle } \\
\text { d'assistant social (arrêté } \\
\text { 13/12/1932) }\end{array}$ & \\
\hline & $\begin{array}{l}\text { Loi du } \\
21 \text { janvier }\end{array}$ & & sur les allocations familiales & \\
\hline & $\begin{array}{l}\text { Loi du } \\
3 \text { juin }\end{array}$ & $\begin{array}{l}\text { le ministère de l'Instruction } \\
\text { publique devient ministere de } \\
\text { l'Éducation nationale }\end{array}$ & & \\
\hline 1933 & $\begin{array}{l}\text { Loi du } \\
14 \text { janvier }\end{array}$ & & $\begin{array}{l}\text { sur la surveillance des } \\
\text { établissements de bienfaisance } \\
\text { privés }\end{array}$ & \\
\hline 1934 & Août & $\begin{array}{l}\text { révolte de la maison d'éducation } \\
\text { surveillée de Bellle-Île-en-Mer, à } \\
\text { l'origine des campagnes de presse } \\
\text { contre les bagnes d'enfants }\end{array}$ & & \\
\hline \multirow[t]{2}{*}{1935} & Mars & $\begin{array}{l}\text { création de la revue Pour l'enfance } \\
\text { "coupable" }\end{array}$ & & \\
\hline & $\begin{array}{l}\text { Décret-loi du } 30 \\
\text { octobre }\end{array}$ & $\begin{array}{l}\text { sur l'assistance éducative et la dépé- } \\
\text { nalisation du vagabondage }\end{array}$ & & \\
\hline 1936 & & & & $\begin{array}{l}\text { législation sur les colonies de } \\
\text { vacances (CEMEA, UFCV) }\end{array}$ \\
\hline
\end{tabular}




\begin{tabular}{|c|c|c|c|c|}
\hline Année & Législation & Justice & Santé & Éducation nationale \\
\hline \multirow[t]{3}{*}{$\begin{array}{l}1936 \\
\text { (suite) }\end{array}$} & $\begin{array}{l}\text { Mai } 1936 \\
\text { Juin } 1937\end{array}$ & & $\begin{array}{l}\text { législation du gouvernement de } \\
\text { Front populaire } \\
\text { - sur les pupilles de l'Assistance } \\
\text { publique } \\
\text { - sur la protection des enfants } \\
\text { assistés } \\
\text { - création du Conseil supérieur de } \\
\text { l'enfance } \\
\text { - création des commissions de } \\
\text { coordination sanitaire et sociale } \\
\text { dans chaque département }\end{array}$ & \\
\hline & $\begin{array}{l}\text { Loi du } \\
9 \text { août }\end{array}$ & & & $\begin{array}{l}\text { l'obligation scolaire est portée } \\
\text { à } 14 \text { ans }\end{array}$ \\
\hline & $\begin{array}{l}\text { Loi du } \\
13 \text { août }\end{array}$ & $\begin{array}{l}\text { réforme de la maison d'éducation } \\
\text { surveillée de Saint-Maurice }\end{array}$ & & \\
\hline \multirow[t]{5}{*}{1937} & $\begin{array}{l}\text { Loi du } \\
10 \text { mars }\end{array}$ & & & $\begin{array}{l}\text { réglementation de l'apprentissage } \\
\text { artisanal }\end{array}$ \\
\hline & $\begin{array}{l}\text { Décret du } \\
22 \text { mai }\end{array}$ & & & $\begin{array}{l}\text { création à titre expérimental des } \\
\text { classes d'orientation }\end{array}$ \\
\hline & $\begin{array}{l}\text { Loi du } \\
12 \text { juillet }\end{array}$ & $\begin{array}{l}\text { réforme de la maison d'éducation } \\
\text { surveillée de Saint-Hilaire }\end{array}$ & & \\
\hline & $\begin{array}{l}\text { Circulaire du } \\
15 \text { septembre }\end{array}$ & & $\begin{array}{l}\text { sur les enfants en dépôt, } \\
\text { en garde, trouvés, moralement } \\
\text { abandonnés }\end{array}$ & \\
\hline & $\begin{array}{l}\text { Décret du } \\
28 \text { septembre }\end{array}$ & $\begin{array}{l}\text { sur le placement des enfants } \\
\text { de Justice }\end{array}$ & & \\
\hline \multirow[t]{2}{*}{1938} & $\begin{array}{l}\text { Avril } 1938 \\
\text { Juillet } 1939\end{array}$ & $\begin{array}{l}\text { collaboration de la direction } \\
\text { de l'Enseignement technique à la } \\
\text { réforme de Saint-Maurice }\end{array}$ & & \\
\hline & $\begin{array}{l}\text { Décret du } \\
17 \text { août }\end{array}$ & $\begin{array}{l}\text { création d'un corps distinct des } \\
\text { personnels d'éducation surveillée } \\
\text { dans l'Administration pénitentiaire }\end{array}$ & & \\
\hline
\end{tabular}




\begin{tabular}{|c|c|c|c|c|}
\hline Année & Législation & Justice & Santé & Éducation nationale \\
\hline $\begin{array}{l}1938 \\
\text { (suite) }\end{array}$ & $\begin{array}{l}\text { Décret du } \\
10 \text { septembre }\end{array}$ & & $\begin{array}{l}\text { sur l'augmentation des frais } \\
\text { d'entretien des mineurs dans les } \\
\text { établissements charitables }\end{array}$ & \\
\hline \multirow[t]{3}{*}{1939} & $\begin{array}{l}\text { Décret-loi du } \\
31 \text { juillet }\end{array}$ & & création du « Code de la famille » & \\
\hline & $\begin{array}{l}\text { Décret-loi du } \\
9 \text { septembre }\end{array}$ & & sur les enfants assistés & \\
\hline & $\begin{array}{l}\text { Décret du } \\
21 \text { septembre }\end{array}$ & & & $\begin{array}{l}\text { création des centres de formation } \\
\text { professionnelle }\end{array}$ \\
\hline \multirow[t]{3}{*}{1940} & $\begin{array}{l}\text { Circulaire du } \\
25 \text { février }\end{array}$ & $\begin{array}{l}\text { les maisons d'éducation surveillée } \\
\text { deviennent IPES (institution } \\
\text { publique d'éducation surveillée) }\end{array}$ & & \\
\hline & $\begin{array}{l}\text { Décret du } \\
31 \text { août }\end{array}$ & $\begin{array}{l}\text { rétablissement de l'interpénétration } \\
\text { entre personnels pénitentiaires } \\
\text { et d'éducation surveillée; les IPES } \\
\text { redeviennent maisons d'éducation } \\
\text { surveillée }\end{array}$ & & \\
\hline & $\begin{array}{l}\text { Loi du } \\
18 \text { septembre }\end{array}$ & & & $\begin{array}{l}\text { le baccalauréat est exigé pour les } \\
\text { instituteurs ; fermeture des écoles } \\
\text { normales, qui deviennent instituts } \\
\text { de formation professionnelle }\end{array}$ \\
\hline \multirow[t]{2}{*}{1941} & $\begin{array}{l}\text { Loi du } \\
15 \text { août } \\
\text { "Loi Carcopino" }\end{array}$ & & & $\begin{array}{l}\text { réforme de l'enseignement } \\
\text { secondaire : } \\
\text { - transformation des écoles } \\
\text { primaires supérieures en collèges } \\
\text { modernes } \\
\text { - suppression de la gratuité } \\
\text { de l'enseignement secondaire }\end{array}$ \\
\hline & Septembre & & $\begin{array}{l}\text { création du Commissariat général } \\
\text { à la famille }\end{array}$ & \\
\hline 1942 & & & $\begin{array}{l}\text { loi de coordination des administra- } \\
\text { tions chargées de l'enfance } \\
\text { déficiente et en danger moral }\end{array}$ & \\
\hline
\end{tabular}




\begin{tabular}{|c|c|c|c|c|}
\hline Année & Législation & Justice & Santé & Éducation nationale \\
\hline \multirow[t]{2}{*}{$\begin{array}{l}1942 \\
\text { (suite) }\end{array}$} & $\begin{array}{l}\text { Circulaire du } \\
21 \text { mars }\end{array}$ & $\begin{array}{l}\text { sur la détention provisoire des } \\
\text { mineurs; création des premiers } \\
\text { centres d'accueil }\end{array}$ & & \\
\hline & $\begin{array}{l}\text { Acte dit « loi du } \\
27 \text { juillet » }\end{array}$ & $\begin{array}{l}\text { sur les tribunaux pour enfants et } \\
\text { adolescents et les centres } \\
\text { d'observation et de triage }\end{array}$ & & \\
\hline \multirow[t]{4}{*}{1943} & Janvier & & $\begin{array}{l}\text { mise en place des premières } \\
\text { Associations régionales de } \\
\text { sauvegarde de l'enfance et de } \\
\text { l'adolescence (ARSEA) } \\
\text { création des premières écoles } \\
\text { d'éducateurs du secteur privé } \\
\text { (Toulouse 1942, Montpellier } \\
\text { 1943...) } \\
\text { ouverture de centres d'observation } \\
\text { et de triage }\end{array}$ & \\
\hline & 25 juillet & & $\begin{array}{l}\text { création du Conseil technique } \\
\text { de l'enfance déficiente en danger } \\
\text { moral }\end{array}$ & \\
\hline & Novembre & $\begin{array}{l}\text { le service de l'Éducation surveillée } \\
\text { à l'intérieur de l'Adminis- } \\
\text { tration pénitentiaire devient une } \\
\text { sous-direction }\end{array}$ & & \\
\hline & & $\begin{array}{l}\text { la direction de l'Administration } \\
\text { pénitentiaire est rattachée au } \\
\text { ministère de l'Intérieur (secrétariat } \\
\text { d'État au maintien de l'ordre) }\end{array}$ & & \\
\hline \multirow[t]{2}{*}{1944} & Loi du 3 juillet & $\begin{array}{l}\text { sur l'enfance malheureuse et } \\
\text { en danger }\end{array}$ & & \\
\hline & Loi du 30 juillet & & $\begin{array}{l}\text { sur l'enfance malheureuse et en } \\
\text { danger (ne sera pas appliquée) }\end{array}$ & \\
\hline
\end{tabular}




\begin{tabular}{|c|c|c|c|c|}
\hline Année & Législation & Justice & Santé & Éducation nationale \\
\hline $\begin{array}{l}1944 \\
\text { (suite) }\end{array}$ & 8 novembre & & & $\begin{array}{l}\text { création de la commission } \\
\text { "Langevin-Wallon " } \\
\text { dont le programme est : } \\
\text { - école unique pour toutes } \\
\text { les structures } \\
\text { - école nouvelle pour la pédagogie }\end{array}$ \\
\hline \multirow[t]{7}{*}{1945} & & & $\begin{array}{l}\text { reconduction et généralisation } \\
\text { des ARSEA }\end{array}$ & \\
\hline & & & $\begin{array}{l}\text { définition du concept d'enfance } \\
\text { inadaptée (travaux du Conseil } \\
\text { technique de l'enfance inadaptée) }\end{array}$ & \\
\hline & $\begin{array}{l}\text { Ordonnance du } \\
2 \text { février }\end{array}$ & sur l'enfance délinquante & & \\
\hline & $\begin{array}{l}\text { Décret du } \\
10 \text { avril }\end{array}$ & $\begin{array}{l}\text { statut des personnels de l'Éducation } \\
\text { surveillée }\end{array}$ & & \\
\hline & $\begin{array}{l}\text { Ordonnance du 1er } \\
\text { septembre }\end{array}$ & $\begin{array}{l}\text { création de la direction de } \\
\text { l'Éducation surveillée, autonome } \\
\text { de l'Administration pénitentiaire } \\
\text { (avec } 8 \text { établissements) }\end{array}$ & & \\
\hline & $\begin{array}{l}\text { Ordonnance du } \\
19 \text { octobre }\end{array}$ & & $\begin{array}{l}\text { réorganisation de l'ensemble des } \\
\text { institutions de Sécurité sociale }\end{array}$ & \\
\hline & $\begin{array}{l}\text { Arrêté du } \\
25 \text { octobre }\end{array}$ & $\begin{array}{l}\text { règlement provisoire des centres } \\
\text { d'observation et des IPES }\end{array}$ & & \\
\hline \multirow[t]{3}{*}{1946} & & & & $\begin{array}{l}\text { création du centre psychopédago- } \\
\text { gique Claude Bernard à Paris }\end{array}$ \\
\hline & Mars & $\begin{array}{l}\text { création de la Revue de } \\
\text { l'Éducation surveillée, qui deviendra } \\
\text { Rééducation en } 1947\end{array}$ & & \\
\hline & Avril & $\begin{array}{l}\text { plan quinquennal de l'Éducation } \\
\text { surveillée (Plan Costa) }\end{array}$ & & \\
\hline
\end{tabular}




\begin{tabular}{|c|c|c|c|c|}
\hline Année & Législation & Justice & Santé & Éducation nationale \\
\hline \multirow[t]{4}{*}{$\begin{array}{l}1946 \\
\text { (suite) }\end{array}$} & $\begin{array}{l}\text { Ordonnance du } \\
26 \text { avril }\end{array}$ & & $\begin{array}{l}\text { assurances sociales étendues à tous } \\
\text { les salariés }\end{array}$ & \\
\hline & Mai & & création de la revue Sawvegarde & \\
\hline & $\begin{array}{l}\text { Loi du } \\
6 \text { août }\end{array}$ & & sur les prestations familiales & \\
\hline & $\begin{array}{l}\text { Décret du } \\
10 \text { décembre }\end{array}$ & tutelle aux prestations familiales & & \\
\hline \multirow[t]{5}{*}{1947} & & $\begin{array}{l}\text { création du SNPES (Syndicat } \\
\text { national des personnels de } \\
\text { l'Éducation surveillée) }\end{array}$ & & \\
\hline & & & $\begin{array}{l}\text { création de l'ANEJI (Association } \\
\text { nationale des éducateurs de jeunes } \\
\text { inadaptés) }\end{array}$ & \\
\hline & & & & $\begin{array}{l}\text { création de la revue Enfance } \\
\text { (Wallon et Le Guillant) }\end{array}$ \\
\hline & 19 juin & & & $\begin{array}{l}\text { remise du plan Langevin-Wallon } \\
\text { qui ne reçoit aucun début } \\
\text { d'application }\end{array}$ \\
\hline & Octobre & & & $\begin{array}{l}\text { premières activités du Centre } \\
\text { national de pédagogie spéciale à } \\
\text { Beaumont (formation des } \\
\text { instituteurs spécialisés) }\end{array}$ \\
\hline \multirow[t]{2}{*}{1948} & & & $\begin{array}{l}\text { création de l'UNARSEA (Union } \\
\text { nationale des associations } \\
\text { régionales de sauvegarde de } \\
\text { l'enfance et de l'adolescence) }\end{array}$ & \\
\hline & & & $\begin{array}{l}\text { création des premières associations } \\
\text { de parents d'enfants inadaptés } \\
\text { et handicapés (ALPERI, Papillons } \\
\text { blancs) }\end{array}$ & \\
\hline
\end{tabular}

